\title{
HEPATITIS
}

\section{New gene IFNL4 is associated with impaired clearance of HCV}

$\mathrm{S}$

cientists report the discovery of a new gene IFNL4 (encoding IFNL4) situated upstream of IFNL3 (also known as $I L 28 B$ ) that is associated with impaired clearance of $\mathrm{HCV}$ infection and response to IFN- $\alpha$ therapy. "The findings indicate that the interferon family has a more complex role in the response to viral infection than was previously recognized," notes author Thomas O'Brien from the NIH, USA, who co-led the transdisciplinary study with Ludmila Prokunina-Olsson, also of the NIH.

A large proportion (up to $80 \%$ ) of individuals infected with $\mathrm{HCV}$ fail to clear the virus and go on to develop chronic $\mathrm{HCV}$ infection, which can lead to cirrhosis or liver cancer. Successful treatment with the currently available therapies largely relies on the $\mathrm{HCV}$ genotype and the genetic make-up of the individual being treated. Previous genome-wide association studies have identified common genetic markers - such as the rs 12979860 and rs8099917 single nucleotide polymorphisms-located on chromosome 19q13.13 near IFNL3 (encoding IFNL3, one of three IFN- $\lambda$ s in the type III IFN family) that are associated with response to HCV treatment and spontaneous clearance of the virus. However, molecular investigations into the function of IFNL3 (IL28B) could not explain these findings.

Prokunina-Olsson led the genomic effort that used next-generation, wholegenome RNA sequencing to provide new insights into the genetic regulation of HCV clearance. Primary human hepatocytes were treated with synthetic double-stranded RNA to mimic HCV infection before RNA sequencing was performed and analysed.

The researchers identified a novel transcribed region upstream of IFNL3. This region harboured a dinucleotide variant (ss469415590) that was found in two alternative forms, either $\Delta \mathrm{G}$ or TT alleles. The one-base deletion in the $\Delta \mathrm{G}$ variant results in a frameshift, which in turn produces the full-length protein they designated IFNL4 (owing to its relatedness and homology to other IFN- $\lambda$ s); the TT

variant does not produce IFNL4.

Strikingly, by analyzing data from HCVinfected patients from several clinical trials (with just over 2,900 participants in total), the authors found that the IFNL4 $\Delta \mathrm{G}$ variant (that is, the presence of fulllength IFNL4 protein) was associated with poorer clearance of $\mathrm{HCV}$ and response to PEG-IFN- $\alpha$ and ribavirin combination therapy than the TT form (that is, absence of IFNL4). Moreover, their analysis determined that $s \$ 469415590$ is a better marker for predicting response to combination therapy and HCV clearance than rs12979860 in African-American individuals; the two markers were of equal standing in European-Americans.

Finally, endogenous expression of IFNL4 (after immunostimulant treatment) in primary human hepatocytes from donors carrying the $\Delta \mathrm{G}$ variant was confirmed. In addition, in vitro studies showed that IFNL4 induces the expression of interferon-stimulated genes (such as STAT1 and OAS1) in a similar pattern to those induced by IFN- $\alpha$ and IFNL3. Interestingly, some of these genes were induced only by IFNL4 (such as CCL5, a marker of HCV-induced liver damage), suggesting that the role of IFNL4 could be distinct from other interferons.

"Whereas other interferon proteins play a positive part in the antiviral response, IFNL4 impairs spontaneous HCV clearance and response to IFN- $\alpha$ based therapy," concludes O'Brien. The authors suggest that IFNL4 could be useful as a novel therapeutic target for $\mathrm{HCV}$ infection. "The IFNL4 $\triangle \mathrm{G}$ variant is more common in people of African ancestry, which helps explain why African American individuals have a lower response to current hepatitis $C$ treatments than patients of Asian or European ancestry," O'Brien adds.

"As IFNL4 was related to poor response to PEG-IFN- $\alpha$ and ribavirin therapy with statistical significance, especially in African American populations, the polymorphism or an expression test [for

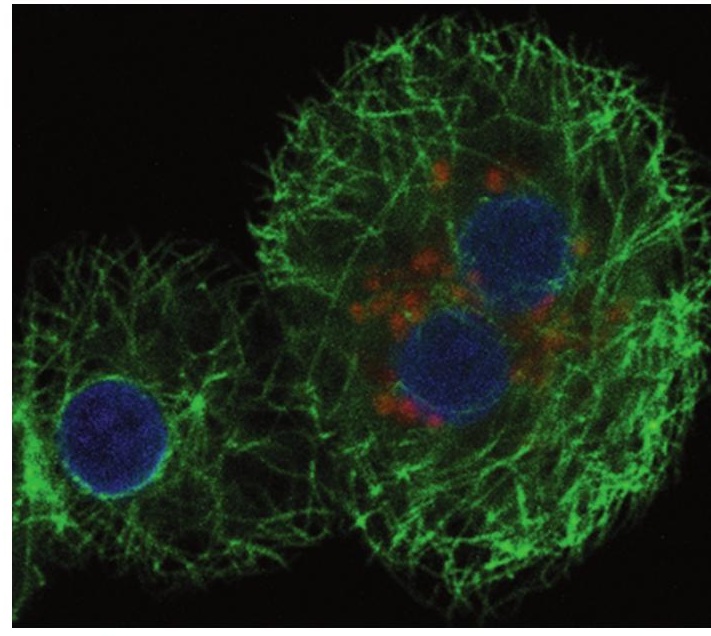

Confocal imaging of primary human hepatocytes from a liver donor heterozygous for the IFNL4 genetic variant. Hepatocytes were imaged after in vitro infection with HCVJFH1 pseudoparticles for $6 \mathrm{~h}$. IFNL4 expression (red) is seen in the cell on the right and was detected with a custom antiIFNL4 monoclonal antibody. Cytoplasm (a-tubulin) and nuclei are stained in green and blue, respectively. Image courtesy of L. Prokunina-Olsson.

IFNL4 itself] could be used to improve the prediction of response to therapy," states Masashi Mizokami from the National Center for Global Health and Medicine, Japan, who was not involved in the study.

Of course, further studies are needed to ascertain the function of IFNL4 and more carefully clarify its role in host response to HCV infection. Mizokami cautions that the findings need to be explored and reproduced in other studies, particularly by comparing IFNL4 expression in liver biopsy and peripheral blood mononuclear cells from patients who have and have not achieved sustained virologic response after treatment. Prokunina-Olsson acknowledges that "the mechanism by which IFNL4 impairs HCV clearance remains unknown and further studies will explore the molecular function of this novel protein".

\section{Katrina Ray}

